PROCEEDINGS OF THE AMERICAN MATHEMATICAL SOCIETY

Volume 124, Number 7, July 1996

\title{
DIFFEOMORPHISMS WITH PERSISTENCY
}

\author{
KAZUHIRO SAKAI
}

(Communicated by Mary Rees)

\begin{abstract}
The $C^{1}$ interior of the set of all diffeomorphisms satisfying Lewowicz's persistency is characterized as the set of all diffeomorphisms satisfying Axiom $\mathrm{A}$ and the strong transversality condition.
\end{abstract}

In [5], Lewowicz introduced a notion of persistency for a homeomorphism of a compact metric space $X$, and it is remarked that persistence is a weaker property than topological stability when $X$ is a manifold. It is also proved there that every pseudo-Anosov map (on a surface) is persistent. The purpose of this paper is to analyze the dynamics of diffeomorphisms having persistency. More precisely we shall prove the following theorem.

Let $M$ be a $C^{\infty}$ closed manifold and let $\operatorname{Diff}(M)$ be the space of $C^{1}$ diffeomorphisms of $M$ endowed with $C^{1}$ topology. We denote by $\mathcal{P}(M)$ the set of all $f \in \operatorname{Diff}(M)$ having persistency.

Theorem. The $C^{1}$ interior of $\mathcal{P}(M)$ in $\operatorname{Diff}(M)$, int $\mathcal{P}(M)$, is characterized as the set of all diffeomorphisms satisfying Axiom $A$ and the strong transversality condition.

It was proved in [7] and [9] respectively that the $C^{1}$ interior of the set of all $f \in \operatorname{Diff}(M)$ having topological stability and the $C^{1}$ interior of the set of all $f \in$ $\operatorname{Diff}(M)$ having the pseudo-orbit tracing property were characterized as the set of all diffeomorphisms satisfying Axiom A and the strong transversality condition. Therefore, if the theorem is established, then these two open sets and $\operatorname{int} \mathcal{P}(M)$ are equal.

Let $\mathcal{E}(M)$ be the set of all expansive diffeomorphisms of $M$. Gerber and Katok [3] proved that if $f$ is a pseudo-Anosov map on a surface $M$ and if $\mathcal{N}^{0}(f)$ is a $C^{0}$ neighborhood of $f$, then there exists a smooth diffeomorphism $g \in \mathcal{N}^{0}(f)$ conjugating to $f$. Thus it can be checked that $g \in \mathcal{E}(M) \cap \mathcal{P}(M)$, and more precisely, the following corollary implies that $g$ belongs to $\mathcal{E}(M) \cap \partial \mathcal{P}(M)$. Here $\partial \mathcal{P}(M)$ denotes the boundary of $\mathcal{P}(M)$ in $\operatorname{Diff}(M)$.

Corollary. $\mathcal{E}(M) \cap \operatorname{int} \mathcal{P}(M)$ is characterized as the set of all Anosov diffeomorphisms.

The corollary is an easy consequence of our theorem. Indeed, since every $f \in$ $\operatorname{Diff}(M)$ satisfying Axiom A and the strong transversality condition is structurally

Received by the editors October 12, 1994 and, in revised form, January 6, 1995.

1991 Mathematics Subject Classification. Primary 54H20, 58F10, 58F15.

Key words and phrases. Persistence, expansive, pseudo-Anosov map, Axiom A, Anosov diffeomorphism.

(C)1996 American Mathematical Society 
stable, if $f \in \mathcal{E}(M)$, then it is Anosov (by [6]). Conversely, if $f \in \operatorname{Diff}(M)$ is Anosov, then $f$ is persistent since $f$ is topologically stable.

Let $d$ be a metric on $M$ which is induced from a Riemannian metric $\|\cdot\|$ on $T M$, and let us denote by $\mathcal{H}(M)$ the set of all homeomorphisms of $M$. We say that $f \in \mathcal{H}(M)$ is persistent if for each $\varepsilon>0$, there is $\delta>0$ such that for every $x \in M$ and $g \in \mathcal{H}(M)$ with $d(f, g)<\delta$, there is $y \in M$ satisfying $d\left(f^{n}(x), g^{n}(y)\right)<\varepsilon$ $(\forall n \in \mathbf{Z})$. The notion is independent of a metric for $M$ and is conjugacy invariant.

Let $\Lambda(f)$ be a hyperbolic set of $f \in \operatorname{Diff}(M)$. For any $\varepsilon>0$ and $x \in \Lambda(f)$, the local stable manifold and the local unstable manifold are denoted by $W_{\varepsilon}^{s}(x, f)$ and $W_{\varepsilon}^{u}(x, f)$ respectively. The stable manifold $W^{s}(x, f)$ and the unstable manifold $W^{u}(x, f)$ of $x \in \Lambda(f)$ are defined by a usual way. Let $f \in \operatorname{Diff}(M)$ satisfy Axiom A. Then the non-wandering set of $f, \Omega(f)$, is a disjoint union of basic sets $\Lambda_{1}(f) \cup \cdots \cup$ $\Lambda_{\ell}(f)$. Recall that the periodic points of $f_{\mid \Lambda_{i}(f)}$ are dense in $\Lambda_{i}(f)(1 \leq i \leq \ell)$ and that for every $x \in M$ there are $p \in \Lambda_{i}(f)$ and $q \in \Lambda_{j}(f)(1 \leq i \neq j \leq \ell)$ such that $x \in W^{s}(p, f) \cap W^{u}(q, f)$. We say that $f$ satisfies the strong transversality condition if for every $x \in M, T_{x} W^{s}(p, f)+T_{x} W^{u}(q, f)=T_{x} M$ for some $p, q \in \Omega(f)$.

Let $P(f)$ denote the set of all periodic points of $f \in \operatorname{Diff}(M)$, and let $\mathcal{F}(M)$ be the set of all $f \in \operatorname{Diff}(M)$ having a $C^{1}$-neighborhood $\mathcal{U}(f) \subset \operatorname{Diff}(M)$ such that every $p \in P(g)(\forall g \in \mathcal{U}(f))$ is hyperbolic. Then such a set was characterized as the set of all diffeomorphisms satisfying Axiom A with no-cycles (see [1, 4]). It is well known that every $f \in \operatorname{Diff}(M)$ satisfying Axiom A and the strong transversality condition is persistent (because $f$ is topologically stable (see [8])). Therefore our theorem follows from the following two propositions.

Proposition A. The $C^{1}$ interior of $\mathcal{P}(M)$, int $\mathcal{P}(M)$, is a subset of $\mathcal{F}(M)$.

Proposition B. Let $f \in \operatorname{Diff}(M)$ satisfy Axiom $A$ with no-cycles. If $f \in$ $\operatorname{int} \mathcal{P}(M)$, then $f$ satisfies the strong transversality condition.

\section{Proof of Proposition A}

Let $f \in \operatorname{int} \mathcal{P}(M)$. To get the conclusion, it is enough to show that every $p \in P(f)$ is hyperbolic. Indeed, if this is established, then for every $C^{1}$ neighborhood $\mathcal{V}(f) \subset$ $\operatorname{int} \mathcal{P}(M)$ of $f$, every $q \in P(g)(\forall g \in \mathcal{V}(f))$ is hyperbolic because $g \in \operatorname{int} \mathcal{P}(M)$. Thus $f \in \mathcal{F}(M)$ is obtained.

Fix a neighborhood $\mathcal{U}(f) \subset \operatorname{int} \mathcal{P}(M)$ of $f$, and by assuming that there is a non-hyperbolic periodic point $p=f^{n}(p)$, we shall derive a contradiction. Here $n>0$ is the prime period of $p$. The tangent space $T_{p} M$ splits into the direct sum $T_{p} M=E_{p}^{u} \oplus E_{p}^{s} \oplus E_{p}^{c}$ where $E_{p}^{u}, E_{p}^{s}$ and $E_{p}^{c}$ are $D_{p} f^{n}$-invariant subspaces corresponding to the absolute values of the eigenvalues of $D_{p} f^{n}$ greater than one, less than one and equal to one, and suppose $E_{p}^{c} \neq 0$. Then, for every $\varepsilon>0$ there exists a linear automorphism $\mathcal{O}: T_{p} M \rightarrow T_{p} M$ such that

$$
\left\{\begin{array}{l}
\|\mathcal{O}-I\| \leq \varepsilon \\
\mathcal{O}\left(E_{p}^{\sigma}\right)=E_{p}^{\sigma} \text { for } \sigma=s, u \text { and } c \\
\text { all eigenvalues of } \mathcal{O} \circ D_{p} f_{\mid E_{p}^{c}}^{n} \text { are of a root of unity, }
\end{array}\right.
$$

where $I: T_{p} M \rightarrow T_{p} M$ is an identity map. By making use of Franks's lemma (see [2, Lemma 1.1]), we can find $\delta_{0}>0$ and $g \in \mathcal{U}(f)$ such that

$$
B_{4 \delta_{0}}\left(f^{i}(p)\right) \cap B_{4 \delta_{0}}\left(f^{j}(p)\right)=\phi \text { for } 0 \leq i \neq j \leq n-1,
$$


(ii) $g(x)=f(x)$ for $x \in\left\{p, f(p), \cdots, f^{n-1}(p)\right\} \cup\left\{M \backslash \cup_{i=0}^{n-1} B_{4 \delta_{0}}\left(f^{i}(p)\right)\right\}$,

(iii) $g(x)=\exp _{f^{i+1}(p)} \circ D_{f^{i}(p)} f \circ \exp _{f^{i}(p)}^{-1}(x)$ for $x \in B_{\delta_{0}}\left(f^{i}(p)\right)(0 \leq i \leq n-2)$,

(iv) $g(x)=\exp _{p} \circ \mathcal{O} \circ D_{f^{n-1}(p)} f \circ \exp _{f^{n-1}(p)}^{-1}(x)$ for $x \in B_{\delta_{0}}\left(f^{n-1}(p)\right)$,

where $B_{\varepsilon}(x)=\{y \in M \mid d(x, y) \leq \varepsilon\}$ for $\varepsilon>0$.

Define $G=\mathcal{O} \circ D_{p} f^{n}$. Then there exists $m>0$ such that $G_{\mid E_{p}^{c}}^{m}$ is an identity map. For a sufficently small $0<\delta_{1}<\delta_{0}$, we have

$$
g_{\mid \exp _{p} T_{p} M\left(\delta_{1}\right)}^{m n}=\exp _{p} \circ G^{m} \circ \exp _{p}^{-1}
$$

where $T_{p} M\left(\delta_{1}\right)=\left\{v \in T_{p} M \mid\|v\| \leq \delta_{1}\right\}$. Put $E_{p}^{c}\left(\delta_{1}\right)=E_{p}^{c} \cap T_{p} M\left(\delta_{1}\right)$. Then it is clear that

$$
g_{\mid \exp _{p} E_{p}^{c}\left(\delta_{1}\right)}^{m n}=i d_{\mid \exp _{p} E_{p}^{c}\left(\delta_{1}\right)} .
$$

Let $v=\left(v_{1}, v_{2}, \cdots, v_{r}\right)\left(r=\operatorname{dim} E_{p}^{c}\right)$ be the representation by components with respect to the fundamental vectors of $\mathbf{R}^{r}=E_{p}^{c}$. Put $\hat{\varepsilon}=\delta_{1} / 8$ and fix any $0<\delta \leq \hat{\varepsilon}$. Let $\varphi: \mathbf{R}^{r} \rightarrow \mathbf{R}^{r}$ be the time-one map given by the vector field

$$
\dot{v}_{i}=\delta^{\prime} \chi\left(v_{1}\right) \cdots \chi\left(v_{r}\right) v_{i}
$$

for $1 \leq i \leq r$. Here $\chi: \mathbf{R} \rightarrow \mathbf{R}$ is a $C^{\infty}$ function $(0 \leq \chi(t) \leq 1)$ such that

$$
\chi(t)= \begin{cases}1 & \text { if }|t| \leq \delta_{1} / 2 \\ 0 & \text { if }|t| \geq 2 \delta_{1} / 3\end{cases}
$$

and $\delta^{\prime}>0$ is a number chosen so that $\|\varphi(v)-v\|<\delta$ for $v \in \mathbf{R}^{r}$ and $\left\|D_{v} \varphi-i d_{\mid \mathbf{R}^{r}}\right\| \leq$ $\left|e^{\delta^{\prime}}-1\right|<\delta$ for $v \in T_{p} M\left(\delta_{1}\right) \cap \mathbf{R}^{r}$. We shall denote by $\tilde{\varphi}: T_{p} M\left(\delta_{1}\right) \rightarrow T_{p} M\left(\delta_{1}\right)$ the extension of $\varphi$ such that $\tilde{\varphi}(v)=\varphi(v)$ for $v \in E_{p}^{c}\left(\delta_{1}\right)$ and $\|\tilde{\varphi}(v)-v\|<\delta$ for $v \in T_{p} M\left(\delta_{1}\right)$. Put

$$
\psi(x)= \begin{cases}\exp _{p} \circ \tilde{\varphi} \circ \exp _{p}^{-1}(x) & \text { if } x \in \exp _{p}\left(T_{p} M\left(\delta_{1}\right)\right), \\ x & \text { otherwise, }\end{cases}
$$

and define $\tilde{g}=\psi \circ g$. Let $\hat{\delta}=\hat{\delta}(g, \hat{\varepsilon})>0$ be a number as in the definition of persistency of $g$. Then $d(\tilde{g}, g)<\hat{\delta}(\tilde{g} \in \mathcal{H}(M))$ for a sufficiently small $\delta$. Take and fix $v \in E_{p}^{c}\left(\delta_{1}\right)$ such that $d\left(\exp _{p}(v), p\right)=2 \hat{\varepsilon}$. Clearly $g^{m n}\left(\exp _{p}(v)\right)=\exp _{p}(v)$ for all $m \in \mathbf{Z}$. On the other hand, it is easy to see that for every $y \in B_{\hat{\varepsilon}}\left(\exp _{p}(v)\right)$, there is $m(y) \in \mathbf{Z}$ such that

$$
d\left(\tilde{g}^{m(y) n}(y), g^{m(y) n}\left(\exp _{p}(v)\right)\right)=d\left(\tilde{g}^{m(y) n}(y), \exp _{p}(v)\right)>\hat{\varepsilon} .
$$

This is a contradiction.

\section{Proof of Proposition B}

Before starting the proof of this propsition, we need some preparation. Throughout this section let $f \in \operatorname{Diff}(M)$ satisfy Axiom A with no-cycles. Take a basic set $\Lambda(f)$ of $f$ and fix $\varepsilon_{0}>0$ sufficiently small. Since $\operatorname{dim} W_{\varepsilon_{0}}^{s}(x, f)=\operatorname{dim} W_{\varepsilon_{0}}^{s}(y, f)$ for $x, y \in \Lambda(f)$, we denote by Ind $\Lambda(f)$ the dimension of $W_{\varepsilon_{0}}^{s}(x, f)$ for $x \in \Lambda(f)$. If $g \in \operatorname{Diff}(M)$ is $C^{1}$ close to $f$, then the number of basic sets $\left\{\Lambda_{i}(g)\right\}$ of $g$ coincides with that of basic sets $\left\{\Lambda_{i}(f)\right\}$ because of $\Omega$-stability of $f$.

The following lemma is induced by Franks's lemma (see [9, Lemma 3] for details). 
Lemma 1. Let $\Lambda_{1}(f)$ and $\Lambda_{2}(f)$ be basic sets for $f$. Suppose that there are $p=$ $f^{n}(p) \in \Lambda_{1}(f)(n>0), q \in \Lambda_{2}(f)$ and $x \in M \backslash \Omega(f)$ satisfying $x \in W^{s}(p, f) \cap$ $W^{u}(q, f)$. Then, for a neighborhood $\mathcal{U}(f) \subset \operatorname{Diff}(M)$ of $f$, there are $0<\varepsilon_{1}<$ $\varepsilon_{0} / 2, g \in \mathcal{U}(f)$ and two distinct basic sets $\Lambda_{1}(g)$ and $\Lambda_{2}(g)$ such that

$$
B_{4 \varepsilon_{1}}\left(f^{i}(p)\right) \cap B_{4 \varepsilon_{1}}\left(f^{j}(p)\right)=\phi \text { for } 0 \leq i \neq j \leq n-1,
$$

(ii)

$$
g(x)= \begin{cases}\exp _{f^{i+1}(p)} \circ D_{f^{i}(p)} f \circ \exp _{f^{i}(p)}^{-1}(x) & \text { if } x \in B_{\varepsilon_{1}}\left(f^{i}(p)\right) \text { for } 0 \leq i \leq n-1, \\ f(x) & \text { if } x \notin \cup_{i=0}^{n-1} B_{4 \varepsilon_{1}}\left(f^{i}(p)\right)\end{cases}
$$

$$
\left\{\begin{array}{l}
g^{n}(p)=p \in \Lambda_{1}(g) \text { and } q \in \Lambda_{2}(g) \\
x \in W^{s}(p, g) \cap W^{u}(q, g) \\
T_{x} W^{s}(p, g)=T_{x} W^{s}(p, f) \text { and } T_{x} W^{u}(q, g)=T_{x} W^{u}(q, f)
\end{array}\right.
$$

Since $f$ satisfies Axiom A, there exist a $D f$-invariant continuous splitting $T_{\Omega(f)} M=E^{s} \oplus E^{u}$ and a constant $0<\lambda<1$ such that

$$
\left\|D f_{\mid E^{s}}^{m}\right\|<\lambda^{m},\left\|D f_{\mid E^{u}}^{-m}\right\|<\lambda^{m}
$$

for all $m \geq 0$. We denote by $E_{x}^{\sigma}$ a fiber of $E^{\sigma}$ at $x \in \Omega(f)$ and put $E_{x}^{\sigma}(\varepsilon)=\{v \in$ $\left.E_{x}^{\sigma} \mid\|v\| \leq \varepsilon\right\}$ for $\varepsilon>0(\sigma=s, u)$. Let $g \in \operatorname{Diff}(M), p=g^{n}(p) \in \Lambda_{1}(g)$ and $\varepsilon_{1}>0$ be given as in Lemma 1 . Then it is easily checked that for $0<\varepsilon \leq \varepsilon_{1}$, we have

$$
\exp _{p}\left(E_{p}^{\sigma}(\varepsilon)\right) \subset W_{\varepsilon_{0}}^{\sigma}(p, g) \text { and } \operatorname{dim} \exp _{p}\left(E_{p}^{\sigma}(\varepsilon)\right)=\operatorname{dim} W_{\varepsilon_{0}}^{\sigma}(p, g)
$$

for $\sigma=s, u$.

Now we shall prove Proposition B. Fix $x \in M \backslash \Omega(f)$ and let $\Lambda_{i}(f)$ and $\Lambda_{j}(f)$ be basic sets of $f$ such that $x \in W^{s}\left(\Lambda_{i}(f), f\right) \cap W^{u}\left(\Lambda_{j}(f), f\right)$. To simplify the proof we assume $i=1$ and $j=2$. If Ind $\Lambda_{1}(f)=\operatorname{dim} M$ or Ind $\Lambda_{2}(f)=0$, then the conclusion of this proposition is clear. Thus we shall prove $T_{x} M=T_{x} W^{s}(x, f)+T_{x} W^{u}(x, f)$ when Ind $\Lambda_{1}(f) \leq \operatorname{dim} M-1$ and $\operatorname{Ind} \Lambda_{2}(f) \geq 1$.

Since $f \in \operatorname{int} \mathcal{P}(M)$ and $\Omega(f)=\overline{P(f)}$, there is $f^{\prime} \in \operatorname{int} \mathcal{P}(M)$ arbitrarily near to $f$ in the $C^{1}$ topology such that

(a) $f(y)=f^{\prime}(y)$ for all $y$ outside of a small neighborhood of $x$,

(b) there are $p=f^{\prime n}(p) \in \Lambda_{1}(f)$ for some $n>0$ and $q \in \Lambda_{2}(f)$ such that $x \in W^{s}\left(p, f^{\prime}\right) \cap W^{u}\left(q, f^{\prime}\right), T_{x} W^{s}\left(p, f^{\prime}\right)=T_{x} W^{s}(x, f)$ and $W^{u}\left(q, f^{\prime}\right)=W^{u}(x, f)$.

By (a), there are basic sets $\Lambda_{i}\left(f^{\prime}\right)(i=1,2)$ for $f^{\prime}$ such that $\Lambda_{i}\left(f^{\prime}\right)=\Lambda_{i}(f)$ since $f$ is $\Omega$-stable. Let us prove $T_{x} M=T_{x} W^{s}\left(p, f^{\prime}\right)+T_{x} W^{u}\left(q, f^{\prime}\right)$. We identify $f^{\prime}$ with $f$ for simplicity, and let $\mathcal{U}(f)$ be a small neighborhood of $f$ such that $\mathcal{U}(f) \subset \mathcal{P}(M)$.

Then, by Lemma 1 there are $g \in \mathcal{U}(f)$ and basic sets $\Lambda_{i}(f)(i=1,2)$ satisfying Lemma 1 (i), (ii) and (iii). Thus $T_{x} W^{s}(p, g)=T_{x} W^{s}(x, f)$ and $W^{u}(q, g)=$ $W^{u}(x, f)$. Pick $\ell>0$ such that $g^{\ell}(x) \in W_{\varepsilon_{1} / 2}^{s}(p, g)$ and $g^{-\ell}(x) \in W_{\varepsilon_{0} / 2}^{u}\left(g^{-\ell}(q), g\right)$, and define

$$
C^{u}\left(g^{\ell}(x)\right)=\text { the connected component of } g^{\ell}(x) \text { in } W^{u}\left(g^{\ell}(q), g\right) \cap B_{\varepsilon_{1}}(p) .
$$

Clearly, $\exp _{p}^{-1}\left(C^{u}\left(g^{\ell}(x)\right)\right) \subset T_{p} M$. 
For a linear subspace $E$ of $T_{p} M$ and $\nu>0$, we write

$$
E_{\nu}\left(g^{\ell}(x)\right)=\left\{v+\exp _{p}^{-1}\left(g^{\ell}(x)\right) \mid v \in E \text { with }\|v\| \leq \nu\right\} .
$$

Then there are a linear subspace $E^{\prime} \subset T_{p} M$ and a number $0<\nu_{0} \leq \varepsilon_{1}$ such that

$$
T_{g^{\ell}(x)} \exp _{p}\left(E_{\nu}^{\prime}\left(g^{\ell}(x)\right)\right)=T_{g^{\ell}(x)} C^{u}\left(g^{\ell}(x)\right)
$$

and $\exp _{p}\left(E_{\nu}^{\prime}\left(g^{\ell}(x)\right)\right) \subset B_{\varepsilon_{1}}(p)$ for $0<\nu \leq \nu_{0}$. Since $g^{\ell}(x) \notin \Omega(g)$, there is $0<\nu_{1} \leq$ $\nu_{0}$ such that $B_{\nu_{1}}\left(g^{\ell}(x)\right) \cap g^{i}\left(B_{\nu_{1}}\left(g^{\ell}(x)\right)\right)=\phi$ for all $i \in \mathbf{Z} \backslash\{0\}$. If $\mathcal{U}(g) \subset \mathcal{U}(f)$ is a neighborhood of $g$, then there are $0<\nu_{2}<\nu_{1} / 4$ and $\varphi \in \operatorname{Diff}(M)$ such that

$$
\left\{\begin{array}{l}
\varphi_{\mid\left(B_{4 \nu_{2}}\left(g^{\ell}(x)\right)\right)^{c}=i d,} \\
\varphi\left(g^{\ell}(x)\right)=g^{\ell}(x), \\
\varphi\left(\exp _{p}\left(E_{\nu_{2}}^{\prime}\left(g^{\ell}(x)\right)\right)\right) \subset C^{u}\left(g^{\ell}(x)\right), \\
\operatorname{dim} \varphi\left(\exp _{p}\left(E_{\nu_{2}}^{\prime}\left(g^{\ell}(x)\right)\right)\right)=\operatorname{dim} C^{u}\left(g^{\ell}(x)\right), \\
g^{\prime} \in \mathcal{U}(g) \text { where } g^{\prime}=\varphi^{-1} \circ g .
\end{array}\right.
$$

We denote $\exp _{p}\left(E_{\nu_{2}}^{\prime}\left(g^{\ell}(x)\right)\right)$ by $\exp _{p}\left(E_{\nu_{2}}^{\prime}\left(g^{\prime \ell}(x)\right)\right)$ because of $g^{\prime \ell}(x)=g^{\ell}(x)$. It is clear that there are two distinct basic sets $\Lambda_{i}\left(g^{\prime}\right)(i=1,2)$ such that $\Lambda_{i}\left(g^{\prime}\right)=\Lambda_{i}(g)$ since $g$ is $\Omega$-stable, and such that

$$
\begin{gathered}
T_{x} W^{\sigma}\left(x, g^{\prime}\right)=T_{x} W^{\sigma}(x, g)(\sigma=s, u), \\
\exp _{p}\left(E_{\nu_{2}}^{\prime}\left(g^{\prime \ell}(x)\right)\right) \subset W^{u}\left(g^{\prime \ell}(q), g^{\prime}\right) \cap B_{\varepsilon_{1}}(p), \\
\operatorname{dim} \exp _{p}\left(E_{\nu_{2}}^{\prime}\left(g^{\prime \ell}(x)\right)\right)=\operatorname{dim} W^{u}\left(q, g^{\prime}\right)=\operatorname{dim} C^{u}\left(g^{\ell}(x)\right) .
\end{gathered}
$$

Lemma 2. Under the above notation, $\exp _{p}\left(E_{\nu_{2}}^{\prime}\left(g^{\prime \ell}(x)\right)\right)$ meets transversely $W_{\varepsilon_{1}}^{s}\left(p, g^{\prime}\right)$ at $g^{\prime \ell}(x)$.

If this lemma is established, then we have $T_{x} M=T_{x} W^{s}(x, f)+T_{x} W^{u}(x, f)$ since $T_{x} W^{\sigma}\left(x, g^{\prime}\right)=T_{x} W^{\sigma}(x, g)=T_{x} W^{\sigma}(x, f)$ for $\sigma=s, u$.

Proof of Lemma 2. Put $C_{\varepsilon}^{u}\left(g^{\prime \ell}(x)\right)=B_{\varepsilon}\left(g^{\prime \ell}(x)\right) \cap g^{\prime 2 \ell}\left(W_{\varepsilon_{0}}^{u}\left(g^{\prime-\ell}(q), g^{\prime}\right)\right)$ for $\varepsilon>$ 0 . Take $0<\tilde{\varepsilon}<\nu_{2}$ such that $C_{\varepsilon}^{u}\left(g^{\prime \ell}(x)\right)$ is the connected component of $g^{\prime \ell}(x)$ in $B_{\tilde{\varepsilon}}\left(g^{\prime \ell}(x)\right) \cap g^{\prime 2 \ell}\left(W_{\varepsilon_{0}}^{u}\left(g^{\prime-\ell}(q), g^{\prime}\right)\right)$ for $0<\varepsilon \leq \tilde{\varepsilon}$, and such that $B_{\tilde{\varepsilon}}\left(g^{\prime \ell}(x)\right) \cap$ $g^{\prime 2 \ell}\left(W_{\varepsilon_{0}}^{u}\left(g^{\prime-\ell}(q), g^{\prime}\right)\right) \subset \exp _{p}\left(E_{\nu_{2}}^{\prime}\left(g^{\prime \ell}(x)\right)\right)$.

Claim. For every $0<\varepsilon \leq \tilde{\varepsilon}$, if $d\left(g^{\prime-i}\left(g^{\prime \ell}(x)\right), g^{\prime-i}(w)\right)<\varepsilon$ for all $i \geq 0$, then $w \in C_{\varepsilon}^{u}\left(g^{\prime \ell}(x)\right)$.

Indeed, it is clear that $d\left(g^{\prime-\ell-i}(x), g^{\prime-2 \ell-i}(w)\right)<\varepsilon \leq \varepsilon_{0} / 2$ for all $i \geq 0$. On the other hand, since $d\left(g^{\prime-\ell-i}(x), g^{\prime-\ell-i}(q)\right)<\varepsilon_{0} / 2(\forall i \geq 0)$,

$$
d\left(g^{\prime-2 \ell-i}(w), g^{\prime-\ell-i}(q)\right) \leq d\left(g^{\prime-2 \ell-i}(w), g^{\prime-\ell-i}(x)\right)+d\left(g^{\prime-\ell-i}(x), g^{\prime-\ell-i}(q)\right)<\varepsilon_{0}
$$

for all $i \geq 0$ and so $g^{\prime-2 \ell}(w) \in W_{\varepsilon_{0}}^{u}\left(g^{\prime-\ell}(q), g^{\prime}\right)$. Thus $w \in C_{\varepsilon}^{u}\left(g^{\prime \ell}(x)\right)=B_{\varepsilon}\left(g^{\prime \ell}(x)\right) \cap$ $g^{\prime 2 \ell}\left(W_{\varepsilon_{0}}^{u}\left(g^{\prime-\ell}(q), g^{\prime}\right)\right)$ since $d\left(g^{\prime \ell}(x), w\right)<\varepsilon$. The claim is proved.

Suppose that $\exp _{p}\left(E_{\nu_{2}}^{\prime}\left(g^{\prime \ell}(x)\right)\right)$ does not meet transversely $W_{\varepsilon_{1}}^{s}\left(p, g^{\prime}\right)$ at $g^{\prime \ell}(x)$. Then there exist $0<\nu_{3}<\min \left\{\tilde{\varepsilon}, \nu_{2} / 2\right\}$ such that for every $\delta>0\left(\delta \ll \nu_{3}\right)$ there is $\psi_{\delta} \in \operatorname{Diff}(M)$ satisfying

$$
\left\{\begin{array}{l}
\left.\psi_{\delta}\right|_{\left(B_{\nu_{3}}\left(g^{\prime \ell}(x)\right)\right)^{c}}=i d \\
d\left(\psi_{\delta}, i d\right)<\delta \\
\psi_{\delta}\left(\exp _{p}\left(E_{\nu_{3} / 2}^{\prime}\left(g^{\prime \ell}(x)\right)\right)\right) \cap W_{\varepsilon_{1}}^{s}\left(p, g^{\prime}\right)=\phi
\end{array}\right.
$$


Fix $0<\varepsilon^{\prime}<\nu_{3} / 2$ and let $\delta^{\prime}=\delta^{\prime}\left(g^{\prime}, \varepsilon^{\prime}\right)>0$ be a number as in the definition of persistency of $g^{\prime}$. Take $\delta>0$ such that $\tilde{g}=g^{\prime} \circ \psi_{\delta} \in \mathcal{H}(M)$ and $d\left(\tilde{g}, g^{\prime}\right)<\delta^{\prime}$. Then, for $g^{\prime}$-orbit $\left\{g^{\prime i}(x)\right\}_{i \in \mathbf{Z}}$ of $x$, there is $y \in B_{\varepsilon^{\prime}}\left(g^{\prime \ell}(x)\right)$ such that $d\left(\tilde{g}^{i}(y), g^{\prime i}\left(g^{\prime \ell}(x)\right)\right)<$ $\varepsilon^{\prime}$ for all $i \in \mathbf{Z}$. By the claim $y \in \exp _{p}\left(E_{\nu_{3} / 2}^{\prime}\left(g^{\prime \ell}(x)\right)\right)$, from which $\tilde{g}(y)=g \circ \psi_{\delta}(y) \notin$

$W_{\varepsilon_{1}}^{s}\left(p, g^{\prime}\right)$. Thus, by the hyperbolicity, $d\left(\tilde{g}^{i}(\tilde{g}(y)), g^{\prime i+1}\left(g^{\prime \ell}(x)\right)\right)>\varepsilon^{\prime}$ for some $i \geq 0$. This is a contradiction.

\section{REFERENCES}

1. N. Aoki, The set of Axiom A diffeomorphisms with no cycle, Bol. Soc. Bras. Mat. 23-New Series (1992), 21-65. MR 94d:58080

2. J. Franks, Necessary conditions for stability of diffeomorphisms, Trans. A.M.S., 158 (1971), 301-308. MR 44:1042

3. M. Gerber and A. Katok, Smooth models of Thurston's pseudo-Anosov maps, Ann. Scient. Éc. Norm. Sup., $4^{e}$ série, t. 15, 1982, 173-204. MR 84e:58056

4. S. Hayashi, Diffeomorphisms in $\mathcal{F}^{1}(M)$ satisfy Axiom A, Ergod. Th. \& Dyn. Sys. 12 (1992), 233-253. MR 94d:58081

5. J. Lewowicz, Persistence in expansive systems, Ergod. Th. \& Dyn. Sys. 3 (1983), 567-578. MR 85m:58140

6. R. Mañé, Expansive Diffeomorphisms, Dynamical Systems-Warwick, 1974, (ed. by A. Manning), Lecture Notes in Math. 468, Springer, 1975, 162-174. MR 58:31263

7. K. Moriyasu, The topological stability of diffeomorphisms, Nagoya Math. J. 123 (1991), 91-102. MR 92g:58067

8. C. Robinson, Stability theorems and hyperbolicity in dynamical systems, Rocky Mountain J. Math., 7 (1977), 425-437. MR 58:13200

9. K. Sakai, Pseudo-orbit tracing property and strong transversality of diffeomorphisms on closed manifolds, Osaka J. Math. 31(1994), 373-386. CMP 95:02

Department of Mathematics, Kanagawa University, Rokkakubashi, Kanagawa-Ku, YOKOHAMA 221, JAPAN

E-mail address: kazsaka@kani.cc.kanagawa.ac.jp 